A. Eliseev, the four cosmonauts "despite a great deal of persuasion from control" had not quietened down until late.

In spite of the excitement of this second linkage, the experimental programme of the mission is continuing to plan. The new crew are involved in an experiment with chlorella and have placed a capsule in the 'Splav' alloying chamber, which is designed to produce alloys of, for example, aluminiumtungsten and monlybdenum-tungsten, which would be impossible except in low or zero gravity conditions. (The particular 'Splav' experiment in which Remek is involved has been named 'Morava' in honour of Czechoslovakia).

However, according to Yakov Ziman of the Soviet Institute of Space Research, the space programme for 1976-80 (including the Comecon Interkosmos programme) is particularly concerned with the earth. Not surprisingly, the over-all plans for Salyut6 have included a considerable geophysical programme which has been going forward steadily throughout the mission. Whereas the Soyuz-22 photographs were "purely scientific", only one-tenth of the Salyut-6 survey is devoted to pure research; the rest are tailored to the requirements of various Ministries, government departments and design organisations.

Much of the data brought back by the crew which visited Salyut at the end of January was at once processed and transmitted to these 'clients', including various organisations involved in opening up the route of the BaikalAmur Mainline railway, and the constructors of the new hydroelectric installations in the highly-seismic vicinity of the Nurek dam.

Other photographic surveys include the monitoring of ocean currents (including oil slicks), and a special study of the glaciers and snow cover of the mountains of Soviet Central Asia. Space photographs are used to monitor glacier movements-a recent TASS announcement claims that over 30,000 "pulsating" glaciers have been discovered in the Pamirs using space photography.

Grechko is apparently a fairly talented antist in the 'space' medium of coloured pencils and felt-tipped pens. This gift is being used in the observation of ocean currents and the aurora borealis. In the case of the ocean observations, there seem to have been some initial difficulties in recording the currents-the colours observed went beyond the scale provided by the oceanologists. Special attention in all such visual observations is being paid to a phenomenan noted by the crew of Soyuz-25-under certain circumstances cloud cover can act as a "gigantic lens" so that waves, for example,

appear considerably magnified.

Other phenomena sketched by Grechko include Venus rises and sunrise over a clear horizon. Some of these sketches were brought back by Soyuz26 and are being studied at the Leningrad Optical Institute. The aurora borealis appearances sketched by Grechko coincided with observations of noctilucent clouds, although, according to Professor Aleksandr I. Lazareev of the Leningrad Optical Institute, it was difficult to establish any link between the two. These were the first space observations of noc-

\title{
Voyager scientific platform jammed
}

VOYAGER 1, the spacecraft launched last summer by the National Aeronautics and Space Administration as part of a joint project to Jupiter and Saturn, has developed an equipment fault which poses a major threat to the scientific success of the mission.

The problem has been caused by a malfunctioning of the scan platform (shaded on diagram) which contains most of the major scientific equipment to be used on the mission, including two television cameras, a cosmic ray detector, a photopolarimeter and ultraviolet and infrared spectrometers.

The platform is designed to rotate along two separate axes-an azimuth axis and an elevation axis - in such a way that the equipment can be pointed at any desired object either in the sky or on the surface of the two planets.

During calibration tests two weeks ago, the movement of the platform on its azimuth axis slowed down to such an extent that it was unable to complete a particular maneouvre in the onehour's computer time alloted, and thus failed to reach the required position.

Further commands issued from earth met with the same response. And al- tilucent clouds in the southern hemisphere, although they had been observed in the northern hemisphere from Salyut-4.

By combining Grechko's observations with data from meterorological rockets launched from the Antarctic Molodezhnaya station it has been deduced that moclilucent clouds have a multi-layer structure, occurring at local temperature minima. A threelayer structure is postulated, distributed at temperatures of -130 to $-150{ }^{\circ} \mathrm{C}$.

Vera Rich though it was initially suggested that the problem was due to an electronic failure in the spacecraft's computer system, it is now thought that the jamming of the platform is due to a mechanical cause.

Scientists at NASA's Jet Propulsion Laboratory in Pasadena, from which the Voyager mission is being directed, are making a hurried analysis of the spacecraft's pre-flight test data, to see if they can find any anomalies in performance which were previous overlooked.

Their intention is to produce a set of diagnostic commands which can be transmitted to the spacecraft. Voyager's reaction to these commands will, it is hoped, enable a fault to be analysed and corrected.

Dr Raymond L. Heacock, programme manager for the Voyager mission, said last week that even if it was impossible to rectify the fault, it would still be possible to get the equipment pointing in desired directions by maneouvring the whole spacecraft. Thus scientific data could still be transmitted back to earth.

However such a procedure would

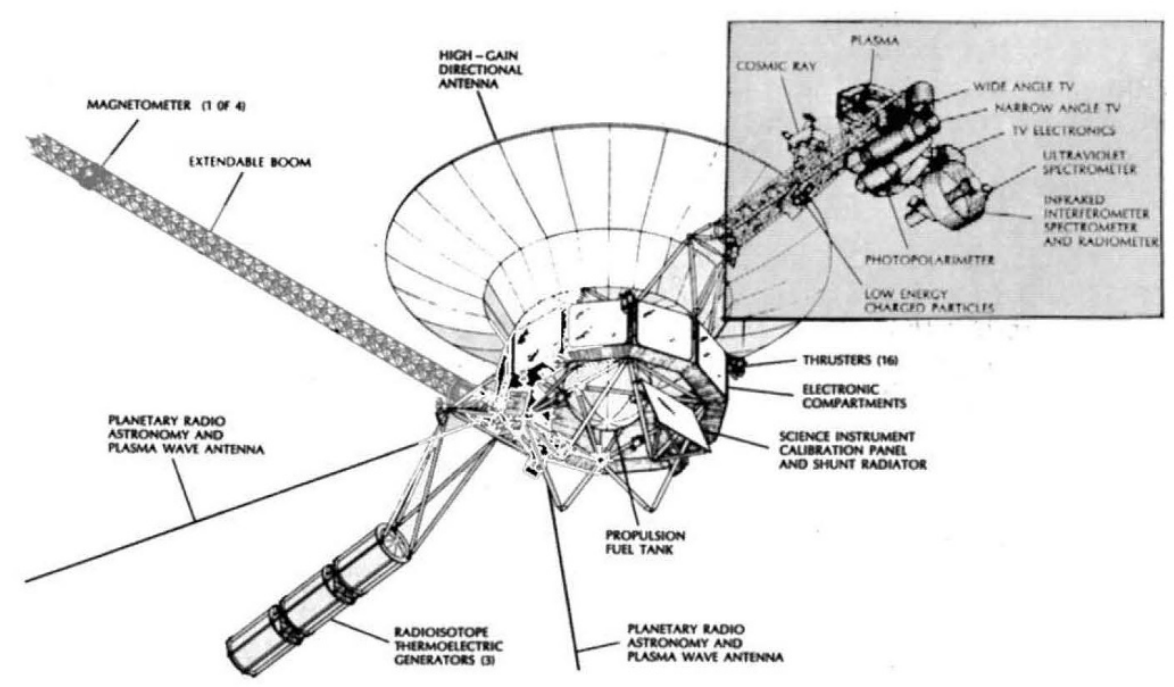


involve a more complex sequence of manoeuvres than initially planned, thus consuming greater quantities of fuel. In addition, the extra time needed to direct the equipment towards a particular target would severely limit the amount of data received.

Voyager 1 was launched last September, and is now over 200 million miles from earth. It is expected to start taking photographs of Jupiter and making spectral scans of the hydrogen cloud surrounding the planet in December, 80 days before reaching the planet.

In late February 1979, eight days from Jupiter, it will begin coverage of the entire planet with its wide-angle camera, while the atmospheric infrared and ultraviolet spectrometers and the photopolarimaters will obtain data on atmospheric composition, temperature variation in the atmosphere, and the possibility of solid particles in the clouds. Observation are, at present, planned to continue at least until April 1979.

A technical problem involving a jammed photopolarimeter filter wheel on board the sister spacecraft Voyager 2 , which was launched 12 days earlier than Voyager 1 but on a slower trajectory, and will not reach Jupiter until July 1979, has already been successfully remedied.

David Dickson

\section{Cassava may lead to mental retardation}

Cassava is the world's seventh most important foodcrop, following the major cereals, potatoes, and yams. Production is in the neighbourhood of 100 million tons, and it is likely to double by the beginning of next century. A major advantage of cassava is that it can grow on poor soil with little rain; it is the principal source of carbohydrates for about 300 million people, most of them in the developing countries of the tropics.

This background underlines the importance of the findings reported by a team of Belgian researchers, headed by Dr André-Marie Ermans of the Department of Radioisotopes at the SaintPierre Hospital, University of Brussels. In a study of the population of Idjwi Island on Lake Kivu, Zaire, they have shown that a steady diet of cassava inhibits iodine uptake by the thyroid gland. When iodine supply is marginal, this can cause endemic goiter, cretinism, and mental retardation.

The Belgian researchers are now following up their study with a campaign in the Ubangi region, in the north-eastern part of Zaire, where goiter is endemic in a population of about one million. They have found that $60-70 \%$ of the inhabitants have endemic goiter, and 1-10\% are affected with cretinism. In addition an unknown number of people suffer from varying degrees of mental retardation. The objective of the campaign, financed by the Belgian government, the Zaïre Institute for Scientific Research, and Canada's International Development Research Centre, is to eradicate goiter and cretinism in the Ubangi region. Some 300000 people have already been "vaccinated" by receiving intramuscular injections of an iodine suspension in oil, that diffuses into the organism over a period of three to seven years; 700,000 more injections are planned.

Congenital hypothyroidism, with its sequels, notably cretinism, is one of the most widespread diseases in the tropics. In the past ten years or so, epidemiological studies in Africa, South America and Asia have revealed that more than 200 million people may be affected by goiter. How much cassava, as a staple food, may contribute to this is yet to be determined.

In the past great emphasis has been laid on research to improve cassava

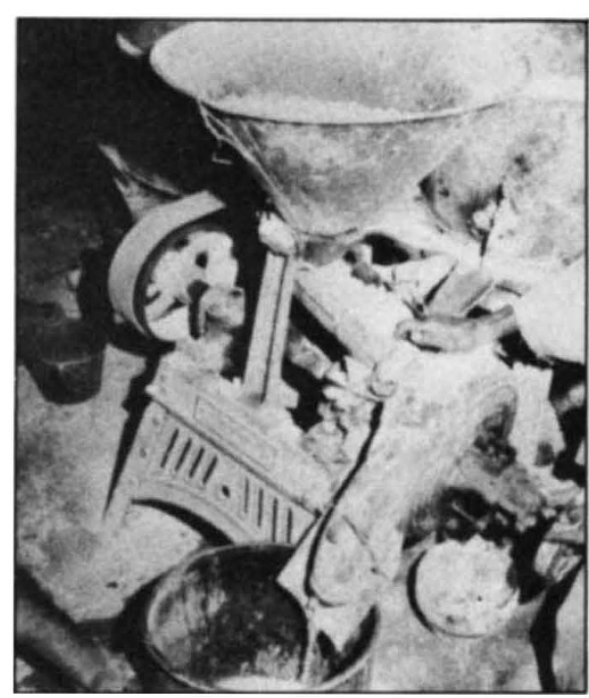

Cassava grinder

productivity and utilisation, but the findings of the Belgian team point to new, and imperative, avenues of research: the prevention of cassavamediated hypothyroidism, better ways of de-toxifying the tuber before it is consumed, and the development of new lines that do not contain the chemicals responsible for this form of toxicity. (Cassava contains cyanogenic gluco- sides; when ingested, these glucosides are detoxified, yielding thiocyanate as a by-product which inhibits iodine uptake by the thyroid.)

These findings, of major importance to developing countries in the tropics, are not without implications for industrialised countries. In Central Europe and in regions along the Mediterranean, where there is limited supply of iodine in food, other vegetables, such as cabbage, may have a similar effect.

François Delange, a Belgian paediatrician, who is participating in the Zaïre programme, has carried out a study in cooperation with Sicilian physicians, and found that high thiocyanate levels are associated with goiter on the island. It is known that in Belgium the iodine content of food is rather low, and tests carried out on 1,800 newborn in Brussels have shown that 14 of them had thyroid insufficiency. If this is not corrected at an early stage, some of these children may become mentally retarded.

The Belgian version of screening for congenital hypothyroidism has certain advantages. It relies on the measurement of the pituitary hormone TSH (thyroid stimulating hormone). A high level indicates transient or permanent thyroid insufficiency. The advantages of the test is that it can be made on a single drop of dried blood, that it can be automated, and is relatively inexpensive (about \$3). It is likely that, as of the end of the year, it will be administered routinely to all newborn children in Belgium.

\section{Alexandre Dorozynski}

\section{Genetically engineered bacterium patented}

The first patent for a genetically engineered microorganism has been granted by the US Court of Patent Appeals to General Electric for a bacterium which degrades crude oil more completely than any bacterium found in nature. A strain of Pseudomonas that can degrade about $60 \%$ of crude oil has been developed in General Electric's New York Laboratories by one of their biologists, Dr A. Chakrabarty.
Many strains of Pseudomonas naturally contain small loops of DNA coding for the breakdown of many of the complex organic compounds making up crude oil, but each strain by itself can only degrade a few of the compounds. Over the past few years, Dr Chakrabarty has constructed strains of Pseudomonas containing several of these DNA plasmids, thus extending the range of compounds that this bacterium can attack. 